\title{
Improving Peripherally Inserted Central Catheter (PICC) care on a Trauma and Orthopaedics ward
}

\author{
Marta Piorkowska, Zahra Al-Raweshidy, Keefai Yeong
}

\begin{abstract}
Peripherally Inserted Central Catheter (PICC) blockage rate was audited over a two month period on the Trauma \& Orthopaedics ward at our District General Hospital. A 70\% (five out of seven) PICC blockage rate was observed. High blockage rates lead to potential treatment complications, delays in delivery of treatment, increase in costs, and reduction in patient satisfaction. The factors contributing to the significant blockage rate include, long and contradictory PICC care guidelines, no information sheets in the patient notes, lack of training and awareness about care of, and flushing of, PICC lines, and lack of accountability for PICC flushing. Our project aimed to achieve a greater rate of PICC patency. We produced one succinct and comprehensive PICC care guideline, carried out staff training sessions, introduced a sticker reminding staff to flush the PICC line after use, and introduced a prescription of weekly heparin saline and PRN saline flushes (for monitoring and accountability). We used questionnaires to assess competency of hospital staff pre-teaching (doctors $6 \%$, nurses $0 \%$ ), and post-teaching (doctors $70 \%$, nurses $38 \%$ ). Blockage rate data post-intervention is pending. Education improved awareness of guidelines amongst staff and we anticipate that the proposed interventions will translate into reduced blockage rates, improving patient outcomes and reducing costs.
\end{abstract}

\section{Problem}

We observed a high (5 out of 7 lines, $70 \%$ ) rate of PICC blockages on the Trauma and Orthopaedics Ward over a two month period.

This led to compromised patient care by delaying drug administration and blood sampling whilst alternative intravenous access was obtained. Blockages were also associated with reduced patient satisfaction, as patients suffered with complications associated with delayed treatment. The cost of treatment increased with the need to replace the PICC. PICC blockages were considered to be time consuming for staff and distracting from other daily duties. Some of the PICCs were blocking repeatedly which was very frustrating for both the staff and patients.

To identify the contributing factors we compared current practice against the local and manufacturer's guidelines and found considerable deviation from guidelines. The following problems were identified:

1. Long and contradictory PICC care guidelines which were difficult to find

2. Lack of information sheets provided in the notes of patients who had a PICC inserted

3. Lack of documentation and accountability regarding flushing PICCs

4. Lack of training and awareness of ward staff regarding care of PICCs

\section{Background}

PICCs are Peripherally Inserted Central Catheters used in patients requiring long term venous access. They may be safer than conventional central lines and can stay in-situ for longer. Indications for use include administration of long term antibiotics, chemotherapy, or total parenteral nutrition (TPN). As with other types of lines, PICCs are subject to complications.

The literature surrounding PICC blockage in the adult population is scarce. The majority of research concentrates on neonatal and paediatric practice, where complications associated with PICCs are well recognised. Barrier et al (1) reported that in their study of an immunocompetent paediatric population, more than $30 \%$ of PICCs developed at least one complication. A neonatal intensive care study undertaken by McCoy et al (2) looked at 1148 PICCs. 164 $(14 \%)$ of these lines were replaced, either because of blockage, or migration of the line.

\section{Baseline Measurement}

We sampled all inpatients with indwelling PICCs over a period of two months on the Trauma \& Orthopaedics (T\&O) ward where we were working as Foundation Year 1 Doctors. Five out of the seven $(70 \%)$ patients with PICCs in situ were affected by line blockage; some on multiple occasions. There was no formal documentation of blockages that had been resolved. Out of the five PICCs affected by blockage, two were unsuccessfully unblocked on the ward and required PICC exchange. This suggests that PICC exchange data would underestimate the scale of the problem.

Subsequently, hospital wide PICC activity was audited. There were 192 inpatient PICCs inserted in the hospital between October 2011 and 2012. 48 of those (25\%) were inserted for T\&O ward patients. During this period, 21 PICCs required exchange (11\%). 8 out of 48 (17\%) PICCs on the T\&O ward required replacement compared with 13 out of 144 PICCs (9\%) on other wards. This further alerted us to suboptimal PICC care on our ward. PICC blockage rates 
(14\%) were comparable with data from a randomised controlled study by Kamala et al (3) which highlights that this problem is not confined to our hospital. Through problem analysis, we identified poor staff training as one of the potential contributors to PICC blockages. We assessed staff competency and confidence levels regarding PICC care using a questionnaire based on PICC care guidelines. Competency was assessed with an informal extended matching questions test; all stems needed to be answered correctly to be awarded a point. Nurses $(n=8)$ all scored $0 \%$ whilst junior doctors $(n=30)$ answered $6 \%$ of questions correctly. This lack of knowledge did not translate into low confidence levels. Staff were asked to rate their confidence level in dealing with PICCs on a scale of $1(0 \%)$ to $5(100 \%)$. Average confidence levels were $65 \%$ amongst nurses and $38 \%$ amongst junior doctors (Table 1 ).

See supplementary file: Table 1.docx

\section{Design}

We asked staff about their potential ideas for change and discuss our planned interventions to gauge feedback. Staff questionnaires highlighted that both nurses and junior doctors were not familiar with flushing regimens. Furthermore, as the flushes were neither prescribed nor signed for, there was no monitoring or accountability for the flushing of PICCs.

Feedback from nursing staff identified that due to shift patterns of work, it was difficult to keep track of when the PICCs were flushed and by whom. Nursing staff reported that this information was rarely handed over. In conjunction with nursing staff, decided to prescribe flushes on the drug chart.

\section{Strategy}

We decided to carry out small-group hands-on teaching tailored to specific staff groups. This was, however, time consuming. We covered accessing PICCs, appropriate care to minimise risk of blockage, infection, and splitting of the line.

Another intervention we designed was a sticker with simple and clear instructions reminding users to flush the PICC after use. Aidememoires already in practice, for example stickers reminding the need to replace a cannula, are in widespread use, and are considered effective.

We collaborated with the radiology and infection control departments to design new guidelines. We advocated prescription of weekly heparin saline and as required (PRN) saline flushes for all patients with PICCs through our guidelines. The guidelines outline appropriate prescription of flushes and indications for PRN flushes. By mandatory prescribing of the flushes on the drug chart, there is uniformity in practice and we believe the flushing will be executed in a more consistent way. Non-adherence to the flushing regimen will be obvious from documentation in the drug chart. We produced two guidelines that, according to staff feedback, was clear, succinct, and easily available. Flow charts illustrated the steps of accessing the PICC line for: blood sampling, drug and fluid administration as well as how to manage a blocked line. One guideline was a short reference guideline available on the wards and in the patient notes. Second was a comprehensive guideline with detailed instructions on care of PICCs. Both guidelines are now available on the Trust Intranet.

\section{Post-Measurement}

We measured the improvement of staff competency and confidence levels in caring for PICCs following a training session (Table 2). Our results were very encouraging. Competence levels increased from $6 \%$ to $70 \%$, (absolute improvement of $64 \%$ ) for junior doctors, and from $0 \%$ to $38 \%$, (absolute improvement of $38 \%$ ) for nurses.

Staff confidence levels followed a similar trend. We observed an increase in confidence levels from $38 \%$ to $66 \%$ (absolute improvement of $28 \%$ ) for junior doctors and from $65 \%$ to $80 \%$ (absolute improvement of $15 \%$ ) for nurses. We are in the process of implementing all the changes described, and we aim to re-audit PICC blockages and exchanges three months post-intervention. We anticipate our interventions will translate into reduced blockage rates, improving patient outcomes, and reducing cost.

Estimating the cost of a single PICC insertion is difficult within the NHS setting. Considering the approximate cost of the PICC, time spent in the interventional radiology suite and staff involved, the cost was estimated to be approximately $£ 1,000$. The cost of the PICC replacement (either exchange or new insertion) was also estimated at approximately $£ 1,000$. There were 21 PICC exchanges undertaken in our hospital within one year (2011-2012). If PICC exchange rate was halved, there is a potential for over $£ 10,000$ of savings per annum. Moreover, there is also a hidden cost; which might be even greater, in time and effort needed to unblock PICCs on the ward. The entire cost of printing the new guidelines and stickers will amount to a fraction of the cost of one PICC exchange.

See supplementary file: Table 2.docx

\section{Lessons and Limitations}

The biggest barrier to change came from staff reluctant to change embedded practice. This was overcome by communicating the need for change and emphasising the benefits of change in decreasing workload long-term.

We have learnt that simple measures can have a significant impact on patient care. In future we would consult individuals involved in the change process earlier on in the project. We also found that our project sometimes was slowed down by other work commitments. We hope to solve this problem by recruiting colleagues to assist us.

We have assumed that the size of the problem of PICC blockages is greater than the PICC exchange rate from our audit on the Trauma \& Orthopaedics ward. It can be argued that a greater sample of wards would be needed to justify such an assertion.

\section{Conclusion}




\section{BMJ Quality Improvement Reports}

There is a lack of PICC care training for staff. Existing information leaflets were unclear and staff were unaware of where to access them. There was little insight from nursing staff as to the degree of variation in current practice from local guidelines and there was no accountability for PICC care. Our interventions should reduce delays in patient care (e.g. delivery of IV antibiotics), improve patient satisfaction and lead to a reduction in cost and overall reduction in workload for staff.

\section{References}

1) Barrier A, Williams DJ, Connelly M, Creech CB. Frequency of peripherally inserted central catheter complications in children. Pediatric Infectious Disease Journal. 2012 May;31(5):519-21.

2) McCoy M, Bedwell S, Noori S. Exchange of peripherally inserted central catheters is associated with an increased risk for bloodstream infection. American Journal of Perinatology. 2011 Jun;28(6):419-24.

3) Kamala F, Boo NY, Cheah FC, Birinder K. Randomized controlled trial of heparin for prevention of blockage of peripherally inserted central catheters in neonates. Acta Paediatrica. 2002;91(12):1350-6. 